\title{
EFECTO DEL EJERCICIO SOBRE LA CINÉTICA DE LA SERIE ERITROCITARIAY LAS CONCENTRACIONES SÉRICAS DE ENZIMAS MUSCULARES EN CABALLOS PURA SANGRE DE CARRERA DE DOS AÑOS DE EDAD
}

\author{
Effect of Exercise on the Kinetics of Erythrocyte Series and Serum Levels \\ of Muscle Enzymes in Two-Year Old Thoroughbred Racehorses
}

\author{
José Luis Collao C. ${ }^{1}$, Olga Lí E..,5, María Vásquez E. ${ }^{2}$, Francisco Suárez A. ${ }^{3}$, \\ Luis Hoyos S. ${ }^{1}$, Pedro Moreno S. ${ }^{4}$, Juan Llamocca G. ${ }^{4}$
}

\section{Resumen}

Se evaluó el efecto del ejercicio que demanda el entrenamiento de los caballos Pura Sangre de Carrera sobre la cinética de la serie eritrocitaria (conteo de glóbulos rojos, hematocrito y hemoglobina) y las enzimas musculares (CK, AST y LDH). Para este fin, se utilizaron 24 animales de dos años de edad (12 machos y 12 hembras) que fueron seleccionados para participar en competencias hípicas de velocidad. Se obtuvieron muestras de sangre de los animales en reposo (T0) y a los 5 minutos (T1), 1 hora (T2) y 24 horas (T3) después del ejercicio en forma mensual y por 5 meses. Las variables recuento de glóbulos rojos y concentración de hemoglobina y hematocrito se incrementaron en el tiempo T1 ( $\mathrm{p}<0.05)$ y solo aumentaron en forma significativa hacia el último mes $(\mathrm{p}<0.05)$. Por otro lado, solo la LDH aumentó significativamente en los tiempos T1 y T2 (p<0.05), mientras que la CK presentó incrementos significativos en el tiempo T2 ( $<<0.05)$. La AST no mostró variaciones significativas en tiempos ni en meses.

Palabras clave: caballo Pura Sangre, valores eritrocíticos, enzimas musculares, CK, AST, $\mathrm{LDH}$, ejercicio

\section{AbStract}

The effect of training in Thoroughbred horses on the kinetic of erythrocytic values (red blood cell count, hemoglobin and hematocrit) and serum muscle enzymes (CK, AST and LDH) was evaluated. Twenty four horses of 2 years of age (12 males and 12 females) were used among a group of horses that were selected to participate in race competitions.

${ }^{1}$ Laboratorio de Patología Clínica, ${ }^{2}$ Laboratorio de Fisiología Animal, ${ }^{3}$ Laboratorio de Medicina Veterinaria Preventiva, Facultad de Medicina Veterinaria, Universidad Nacional Mayor de San Marcos, Lima

${ }^{4}$ Hipódromo de Monterrico, Jockey Club del Perú, Lima

${ }^{5}$ E-mail: olgalie@hotmail.com

Recibido: 15 de febrero de 2012

Aceptado para publicación: 29 de mayo de 2013 
Blood samples were monthly taken before exercise (T0), and 5 minutes (T1), 1 hour (T2) and 24 hours (T3) after exercise during five months. The RBC count, hemoglobin concentration and hematocrit significantly increased in $\mathrm{T} 1(\mathrm{p}<0.05)$ and in the fifth month $(p<0.05)$. Also, LDH increased at T1 and T2 $(p<0.05)$, while CK increases in T2 $(p<0.05)$. AST did not show significant variation through collection times or months of training.

Key words: Throroughbred horse, erythrocytic values, muscular enzymes, CK, AST, LDH, exercise

\section{INTRODUCCIÓN}

La hípica es una industria popular y multimillonaria a nivel mundial, donde los caballos son sometidos a grandes esfuerzos físicos que los predispone a sufrir claudicaciones, y que en muchos casos pueden ser la respuesta a una condición de entrenamiento deficiente (Hinchcliff et al., 2009). Por este motivo, los profesionales dedicados a la hípica muestran especial interés en encontrar métodos de evaluación para estimar la respuesta del equino al entrenamiento (Cofré, 2005).

Diversos investigadores han estudiado los cambios hematológicos producidos durante el ejercicio, que comprometen a la serie roja y blanca, a fin de evaluar el estado normal de los individuos y las adaptaciones al ejercicio (Álvarez, 2006). Adicionalmente, se ha empleado la determinación de la actividad de algunas enzimas que regulan las vías metabólicas aeróbicas y anaeróbicas para evaluar la capacidad metabólica y la adaptabilidad del músculo esquelético al ejercicio (Merino et al., 1998).

A pesar de la información existente del efecto del ejercicio sobre parámetros hematológicos, no se dispone de mucha información para el caso de caballos jóvenes Pura Sangre de Carrera (PSC) (Cötelioglu et al., 2001); no obstante, el análisis de las modificaciones de estos parámetros posejercicio puede permitir generar una base de datos específica para las condiciones en que se desarrollan los deportes hípicos en cada país (Perrone et al., 2006).
En el Perú, los caballos PSC de dos años de edad son trasladados de los criaderos al hipódromo donde son sometidos a un entrenamiento que consta de seis etapas: amansada o etapa previa, educación en torno, primeros galopes en pista, entrenamiento con trabajos cortos, entrenamiento desde el partidor, y primera carrera o debut. De no presentarse alteraciones musculoesqueléticas o sanitarias, estos animales podrían tener su primera carrera a los 150 días del inicio del entrenamiento (P. Moreno, Lima, comunicación personal).

Por tal motivo, el presente estudio tuvo por objetivo determinar los valores pre y posejercicio de la serie ertitrocitaria (recuento de eritrocitos, concentración de hemoglobina, y paquete celular o hematocrito) y de las concentraciones séricas de enzimas (CK, AST y LDH) en caballos PSC de dos años de edad durante los primeros cinco meses de entrenamiento.

\section{Materiales y Métodos}

Se trabajó con 24 caballos PSC (12 machos y 12 hembras) de dos años de edad del Hipódromo de Monterrico, Lima, Perú. Estos animales no habían participado en competencias hípicas y se encontraban en aparente buen estado de salud.

El esquema de toma de muestras estuvo relacionado al comportamiento de la serie eritrocitaria y enzimas musculares, dado que estas últimas realizan picos en distintos momentos posteriores al ejercicio. Así, a cada 
animal se le extrajo cuatro juegos de muestras de sangre (T0, T1, T2, T3) cada 30 días durante un periodo de cinco meses o hasta el día de su primera carrera. Las muestras se colectaron según el siguiente esquema:

- T0: En reposo, previo a la salida al calentamiento

- T1: 5 minutos después de finalizado el ejercicio

- $\quad$ T2: 1 hora después de finalizado el ejercicio

- T3: 24 horas después de finalizado el ejercicio
La muestra de sangre $(9 \mathrm{ml})$ se extrajo por punción de la vena yugular y se dividió en dos tubos rotulados con la identificación del animal. El tubo correspondiente a la evaluación de la serie eritrocitaria $(3 \mathrm{ml})$ contenía EDTA y el tubo para determinar las concentraciones séricas de las enzimas musculares (6 ml) fue sin anticoagulante para la obtención de suero.

Las muestras de sangre fueron trasladadas en una caja térmica con refrigerante al Laboratorio de Patología Clínica de la Facultad de Medicina Veterinaria de la Univer-

Cuadro 1. Recuento de glóbulos rojos $\left(\mathrm{x} 10^{6} / \mu \mathrm{l}\right)$, concentración de hemoglobina $(\mathrm{g} / \mathrm{dl})$ y hematocrito (\%) en caballos Pura Sangre de Carrera de dos años de edad durante el periodo de preparación para su primera carrera (promedio y desviación estándar)

\begin{tabular}{|c|c|c|c|c|c|}
\hline \multirow{2}{*}{ Mes } & \multirow{2}{*}{$\begin{array}{c}\text { Serie } \\
\text { eritrocitaria }\end{array}$} & \multicolumn{4}{|c|}{ Muestra $^{1}$} \\
\hline & & T0 & $\mathrm{T} 1$ & $\mathrm{~T} 2$ & T3 \\
\hline \multirow[t]{3}{*}{1} & G. rojos & $8.97 \pm 1.1^{\mathrm{a}^{1}}$ & $11.42 \pm 1.3^{\mathrm{b}}$ & $8.81 \pm 0.9^{\mathrm{a}}$ & $9.15 \pm 1.1^{\mathrm{a}}$ \\
\hline & Hemoglobina & $12.2 \pm 1.6^{\mathrm{a} 1}$ & $15.1 \pm 1.6^{\mathrm{b}}$ & $12.3 \pm 1.4^{\mathrm{a}}$ & $12.7 \pm 1.3^{\mathrm{a}}$ \\
\hline & Hematocrito & $35.8 \pm 3.6^{\mathrm{a} 1}$ & $44.4 \pm 4.8^{\mathrm{b}}$ & $35.7 \pm 2.8^{\mathrm{a}}$ & $36.8 \pm 3.7^{\mathrm{a}}$ \\
\hline \multirow[t]{3}{*}{2} & G. rojos & $9.09 \pm 0.8^{\mathrm{a}^{1}}$ & $11.46 \pm 1.5^{\mathrm{b}}$ & $9.18 \pm 0.8^{\mathrm{a}}$ & $8.95 \pm 1.0^{\mathrm{a}}$ \\
\hline & Hemoglobina & $12.4 \pm 1.0^{\mathrm{a} 1}$ & $14.9 \pm 1.9^{b}$ & $12.5 \pm 1.3^{\mathrm{a}}$ & $12.5 \pm 1.2^{\mathrm{a}}$ \\
\hline & Hematocrito & $36.2 \pm 2.8^{\mathrm{a} 1}$ & $43.8 \pm 5.0^{\mathrm{b}}$ & $36.3 \pm 3.0^{\mathrm{a}}$ & $35.9 \pm 3.6^{\mathrm{a}}$ \\
\hline \multirow[t]{3}{*}{3} & G. rojos & $8.99 \pm 0.8^{\mathrm{a} 1}$ & $11.41 \pm 1.4^{\mathrm{b}}$ & $9.27 \pm 1.3^{\mathrm{a}}$ & $9.19 \pm 1.1^{\mathrm{a}}$ \\
\hline & Hemoglobina & $12.3 \pm 1.3^{\mathrm{a} 1}$ & $15.1 \pm 1.8^{\mathrm{b}}$ & $12.5 \pm 1.4^{\mathrm{a}}$ & $12.6 \pm 1.3^{\mathrm{a}}$ \\
\hline & Hematocrito & $35.7 \pm 3.3^{\mathrm{a} 1}$ & $44.0 \pm 5.2^{\mathrm{b}}$ & $36.5 \pm 4.1^{\mathrm{a}}$ & $36.4 \pm 3.7^{\mathrm{a}}$ \\
\hline \multirow[t]{3}{*}{4} & G. rojos & $9.45 \pm 1.4^{\mathrm{a}^{1}}$ & $11.32 \pm 1.7^{\mathrm{b}}$ & $9.31 \pm 1.2^{\mathrm{a}}$ & $9.60 \pm 1.3^{\mathrm{a}}$ \\
\hline & Hemoglobina & $12.5 \pm 1.7^{\mathrm{a} 1}$ & $14.7 \pm 2.2^{\mathrm{b}}$ & $12.8 \pm 1.6^{\mathrm{a}}$ & $12.9 \pm 1.7^{\mathrm{a}}$ \\
\hline & Hematocrito & $37.0 \pm 4.9^{\mathrm{a} 1}$ & $43.3 \pm 6.2^{\mathrm{b}}$ & $37.0 \pm 3.9^{\mathrm{a}}$ & $35.5 \pm 4.5^{\mathrm{a}}$ \\
\hline \multirow[t]{3}{*}{5} & G. rojos & $9.65 \pm 1.1^{\mathrm{a}^{2}}$ & $11.39 \pm 1.8^{\mathrm{b}}$ & $9.31 \pm 1.1^{\mathrm{a}}$ & $9.48 \pm 1.2^{\mathrm{a}}$ \\
\hline & Hemoglobina & $13.5 \pm 1.2^{\mathrm{a} 2}$ & $15.3 \pm 2.2^{\mathrm{b}}$ & $13.1 \pm 1.2^{\mathrm{a}}$ & $13.3 \pm 1.6^{\mathrm{a}}$ \\
\hline & Hematocrito & $38.7 \pm 3.3^{\mathrm{a} 2}$ & $44.1 \pm 6.0^{\mathrm{b}}$ & $37.5 \pm 3.2^{\mathrm{a}}$ & $37.9 \pm 3.9^{\mathrm{a}}$ \\
\hline
\end{tabular}

${ }^{1}$ T0: En reposo previo a la salida al calentamiento; T1, T2 y T3: 5 minutos, 1 hora y 24 horas después de finalizado el ejercicio, respectivamente

$a, b, c$ Superíndices (letras) diferentes dentro de líneas indican diferencias estadísticas $(p<0.05)$

1,2 Superíndices diferentes en T0 y dentro de cada variable eritrocitaria indican diferencias estadísticas $(\mathrm{p}<0.05)$ 
Cuadro 2. Lactato deshidrogenasa (LDH, UI/L), Aspartato amino transfersa (AST, UI/L) y creatina quinasa (CK, UI/L) en caballos Pura Sangre de Carrera de dos años de edad durante el periodo de preparación para su primera carrera (promedio y desviación estándar)

\begin{tabular}{clcccc}
\hline \multirow{2}{*}{ Mes } & Enzimas & \multicolumn{4}{c}{ Muestra $^{1}$} \\
\cline { 3 - 6 } & musculares & T0 & T1 & T2 & T3 \\
\hline \multirow{4}{*}{1} & LDH & $771.6 \pm 188^{\mathrm{a}^{1}}$ & $864.4 \pm 238^{\mathrm{b}}$ & $869.9 \pm 296^{\mathrm{b}}$ & $748.5 \pm 225^{\mathrm{a}}$ \\
& AST & $571.6 \pm 374^{\mathrm{a} 1}$ & $599.3 \pm 358^{\mathrm{a}}$ & $620.5 \pm 436^{\mathrm{a}}$ & $627.6 \pm 515^{\mathrm{a}}$ \\
& CK & $246.3 \pm 95^{\mathrm{a}}$ & $357.4 \pm 358^{\mathrm{a}}$ & $1080.3 \pm 3151^{\mathrm{b}}$ & $247.7 \pm 125^{\mathrm{a}}$ \\
\multirow{4}{*}{2} & LDH & $811.0 \pm 299^{\mathrm{a}^{\mathrm{a}}}$ & $927.8 \pm 376^{\mathrm{b}}$ & $903.8 \pm 340^{\mathrm{b}}$ & $711.5 \pm 215^{\mathrm{a}}$ \\
& AST & $725.5 \pm 624^{\mathrm{a} 1}$ & $716.3 \pm 554^{\mathrm{a}}$ & $756.0 \pm 605^{\mathrm{a}}$ & $674.3 \pm 477^{\mathrm{a}}$ \\
& CK & $541.0 \pm 894^{\mathrm{a} 1}$ & $648.9 \pm 983^{\mathrm{a}}$ & $945.3 \pm 1247^{\mathrm{b}}$ & $299.5 \pm 211^{\mathrm{a}}$ \\
& LDH & $758.2 \pm 324^{\mathrm{a} 1}$ & $805.4 \pm 290^{\mathrm{b}}$ & $791.3 \pm 304^{\mathrm{b}}$ & $715.7 \pm 301^{\mathrm{a}}$ \\
& AST & $618.3 \pm 552^{\mathrm{a} 1}$ & $653.6 \pm 644^{\mathrm{a}}$ & $655.6 \pm 625^{\mathrm{a}}$ & $624.2 \pm 559^{\mathrm{a}}$ \\
& CK & $228.8 \pm 98^{\mathrm{a} 1}$ & $283.8 \pm 126^{\mathrm{a}}$ & $412.4 \pm 386^{\mathrm{b}}$ & $247.0 \pm 138^{\mathrm{a}}$ \\
& LDH & $713.0 \pm 338^{\mathrm{a}^{1}}$ & $765.8 \pm 326^{\mathrm{b}}$ & $733.1 \pm 307^{\mathrm{b}}$ & $673.5 \pm 258^{\mathrm{a}}$ \\
& AST & $556.1 \pm 489^{\mathrm{a} 1}$ & $541.7 \pm 425^{\mathrm{a}}$ & $540.6 \pm 445^{\mathrm{a}}$ & $550.4 \pm 449^{\mathrm{a}}$ \\
& CK & $328.3 \pm 456^{\mathrm{a} 1}$ & $440.8 \pm 734^{\mathrm{a}}$ & $663.1 \pm 1090^{\mathrm{b}}$ & $268.8 \pm 168^{\mathrm{a}}$ \\
& LDH & $670.1 \pm 183^{\mathrm{a} 1}$ & $726.6 \pm 193^{\mathrm{b}}$ & $722.8 \pm 290^{\mathrm{b}}$ & $674.2 \pm 255^{\mathrm{a}}$ \\
5 & AST & $668.1 \pm 593^{\mathrm{a} 1}$ & $685.7 \pm 637^{\mathrm{a}}$ & $642.5 \pm 496^{\mathrm{a}}$ & $651.2 \pm 549^{\mathrm{a}}$ \\
& CK & $324.3 \pm 281^{\mathrm{a} 1}$ & $401.4 \pm 413^{\mathrm{a}}$ & $866.6 \pm 1447^{\mathrm{b}}$ & $330.8 \pm 330^{\mathrm{a}}$ \\
\hline
\end{tabular}

${ }^{1}$ T0: En reposo previo a la salida al calentamiento; T1, T2 y T3: 5 minutos, 1 hora y 24 horas después de finalizado el ejercicio, respectivamente $a, b$ Superíndices (letras) diferentes dentro de líneas indican diferencias estadísticas $(p<0.05)$

1,2 Superíndices diferentes en T0 y dentro de cada variable enzimática indican diferencias estadísticas $(p<0.05)$

sidad Nacional Mayor de San Marcos, Lima, y fueron procesadas de inmediato. El suero se obtuvo por centrifugación a $4000 \mathrm{rpm}$ por 10 minutos.

En las muestras de sangre entera se determinó: Recuento de glóbulos rojos, Concentración de hemoglobina y Paquete celular o hematocrito, mientras que en las muestras de suero se determinó la concentración de enzimas musculares (Lactato Deshidrogenasa o LDH, Aspartato Amino Transferasa o AST y Creatina Quinasa o CK).
Para el análisis de los datos se empleó estadística descriptiva utilizando la media aritmética como medida de tendencia central y la desviación estándar como medida de dispersión. Posteriormente, los efectos lineales y cuadráticos de los valores eritrocíticos y las concentraciones de enzimas musculares se determinaron mediante el test de análisis de varianza y el test de análisis de varianza posestimación. Adicionalmente, los efectos lineales y cuadráticos fueron modelados mediante la prueba del «Modelo Mixto Lineal» (Searle et al., 1992). Finalmente, se genera- 
ron variables «dummy» para determinar diferencias entre meses y entre tiempos de muestreo en cada mes. Los datos fueron procesados mediante el paquete estadístico STATA 10.0 con un nivel de confianza del $95 \%$ y un nivel de significancia de $\mathrm{p}<0.05$.

\section{Resultados y Discusión}

Los valores de la serie eritrocitaria estuvieron debajo del rango normal para equinos PSC (Lording, 2008) en el primer mes del estudio (Cuadro 1), debido en parte a que los animales provienen de criaderos donde la alimentación se basa en pasturas y no en base a suplementos nutricionales. Además, el incremento paulatino de los valores se debió al estímulo eritropoyético del ejercicio como respuesta adaptativa al incremento de las cargas y tiempo de ejercicio (Gómez et al., 2004). Por otro lado, el aumento de los valores en el T1 fue causado por el estímulo adrenérgico del ejercicio que genera una contracción del bazo.

La variación en las concentraciones séricas de las enzimas musculares estuvo relacionada al tiempo y carga de trabajo (Cuadro 2). La LDH se incrementó hacia el segundo mes para posteriormente disminuir, lo que demostró adaptación al ejercicio; asimismo, se incrementó en los tiempos $\mathrm{T} 1$ y T2, lo que concuerda con Cötelioglu et al. (2001).

La CK presentó valores superiores a los encontrados por Gómez et al. (2004), posiblemente debido a las mayores cargas de trabajo de los PSC en comparación a los caballos de salto. Además, mostró un incremento marcado en T2, lo cual está correlacionado a su vida media en sangre y al tipo de entrenamiento realizado, normalizándose los valores a las 24 horas, indicando que el esfuerzo realizado fue controlado. Por otro lado, la AST no reflejó diferencias ni entre tiempos ni entre meses de muestreo, dado que es una enzima inespecífica, aunque junto a las anteriores puede ayudar en el diagnóstico de afecciones músculo-esqueléticas.
Las variables que mostraron un mayor cambio fueron los valores de la serie eritrocitaria, los cuales se incrementaron de manera significativa hacia el último mes demostrando la adaptación al ejercicio.

\section{Conclusiones}

- El ejercicio produjo un aumento paulatino de los valores eritrocitarios en los caballos Pura Sangre de Carrera de dos años de edad, especialmente al quinto mes del periodo de entrenamiento $(\mathrm{p}<0.05)$.

- Los valores eritrocitarios tuvieron un aumento significativo inmediatamente después de finalizado el ejercicio $(\mathrm{p}<0.05)$, los cuales volvieron al rango normal en el espacio de una hora.

- La enzima LDH tuvo un aumento significativo cinco minutos después del ejercicio, en tanto que la enzima CK lo presentó a la hora de realizado el ejercicio $(\mathrm{p}<0.05)$.

- Las tres enzimas presentaron variaciones no significativas durante los cinco meses del periodo de entrenamiento.

\section{LitTeratura Cittada}

1. Álvarez J. 2006. Efecto de dos ejercicios diferentes sobre el hemograma, cortisol y proteínas plasmáticas en equinos mestizos fina sangre inglés entrenados para participar en pruebas de resistencia. Tesis de Médico Veterinario. Chillán, Chile: Facultad de Medicina Veterinaria, Universidad de Concepción. $56 \mathrm{p}$.

2. Cofré S. 2005. Determinación de los parámetros fisiológicos, ácido láctico y enzimas en equinos de silla francés durante el segundo año de entrenamiento para competencia ecuestre. Tesis de Médico Veterinario. Chillán, Chile: Facultad de Medicina Veterinaria, Universidad de Concepción. 49 p. 
3. Cötelioglu $\ddot{U}$, Arslan M, Matar E, Bakirel U, Özcan M, Tosun C. 2001. The effects of physical exercise on some physiological parameters, plasma $\mathrm{CK}$ and LDH levels in horses that are breeded as race horses. J Fac Vet Med Istanbul Univ 27: 609-615.

4. Gómez C, Petrón P, Andaur M, Pérez $R$, Matamoros R. 2004. Medición postejercicio de variables fisiológicas, hematológicas y bioquímicas en equinos de salto holsteiner. Rev Cien FCV-LUZ 14: 244-253.

5. Hinchcliff K, Morley P, Guthrie A. 2009. Efficacy of furosemide for prevention of exercise-induced pulmonary hemorrhage in Thoroughbred racehorses. JAVMA 235: 76-82.
6. Lording P. 2008. Erythrocytes. Vet Clin Equine 24: 225-237.

7. Merino V, Islas A, López-Rivero J, Mora G, Quezada M, López J, Reyes J. 1998. Características metabólicas del músculo Gluteusmedius de equinos mestizos con aptitud de tiro. Arch Med Vet 30: 125-130.

8. Perrone GM, Caviglia JF, Pérez A, Fidanza M, Marquez A, Catelli JL, González G 2006. Cambios en las variables fisiológicas en equinos compitiendo en una prueba combinada. An Vet 22: 35-42.

9. Searle S, Casella G, McCullogh C. 1992. Modelos lineales de efectos mixtos. En: Searle S, Casella G, McCullogh C (eds). Componentes de la varianza. EEUU: John Wiley \& Sons. p 122-159. 